Conclusion Our findings show that monochorionicity and nulliparity are associated with adverse perinatal outcomes in twin pregnancies, and confirm that these pregnancies warrant close antenatal surveillance.

\section{PP.52 NATIONAL REVIEW OF POSTMORTEM RATES IN IRELAND - ARE WE TO BLAME?}

doi:10.1136/archdischild-2013-303966.330

${ }^{1} \mathrm{~K}$ McNamara, ${ }^{2} \mathrm{~A}$ Heazell, ${ }^{1} \mathrm{~K}$ O'Donoghue. ${ }^{1}$ Anu Research Center, Department Of Obstetrics and Gynaecology, Cork University Maternity Hospital, Cork, Ireland; ' ${ }^{2}$ Maternal and Fetal Research Group, University Of manchester, Manchester, UK

One in 200 babies in the Republic of Ireland is stillborn. Investigating the cause of stillbirth helps gives the clinician valuable information in relation to managing a subsequent pregnancy. A PM is the most useful and informative investigation that can be performed in the postnatal period.

PM uptake rates in Ireland are low, circa 50\%. There are few guidelines for staff on how to broach this issue and it is possible that individual clinicians' attitudes to PM affect the uptake rates.

We conducted a questionnaire study examining the attitudes of midwives and obstetricians in Ireland to perinatal PM. This questionnaire has been validated by the ISA, ANZSA and also by UKSands.

In total 117 questionnaires were completed. Obstetricians made up $44 \%$ of respondents - the rest were midwives. $17 \%$ of respondents never gave information to parents about PMs. Of those who did, only $37 \%$ did so at the time of diagnosis. The majority of respondents $(76 \%)$ were dissatisfied with the quality of training received in how to counsel for PM. $46 \%$ of respondents were satisfied with the quality of information materials available in counselling parents re PM. $83 \%$ felt that a clinical guideline would be helpful to assist parents in decision making.

To summarise, there is a wide variation among health care staff in the current knowledge and clinical practise relating to stillbirth and consenting for PM. We anticipate that by introducing a standardised guideline and improving staff education that our PM rates will improve dramatically.

\section{PP.53 DOMESTIC VIOLENCE AND TERMINATION OF PREGNANCY: A SYSTEMATIC REVIEW OF THE LITERATURE}

doi:10.1136/archdischild-2013-303966.331

1,2M Hall, 'LC Chappell, 1.2BL Parnell, 'S Bewley. 'Women's Health Academic Division, St Thomas' Hospital, London, UK; 'uyy's, King's and St Thomas' Medical School, King's College London, London, UK

Introduction Domestic violence (DV) during pregnancy is common and correlated with maternal and perinatal morbidity and mortality, but less is known about the association with termination of pregnancy (TOP). The aim of this research was to synthesise current evidence on the association between DV and TOP.

Methods A systematic review was undertaken, with a structured search of four databases and reference list screening. Screening of titles and abstracts (241 papers excluding duplicates) was carried by two authors and selected papers were read in full (71). Inclusion criteria were peer-reviewed publication with defined methodology studying TOP and an aspect of DV. Formal data extraction and CASP scoring of 50 quantitative and 4 qualitative studies was undertaken by two authors. PRISMA guidelines were followed.

Results $91 \%$ of studies from 19 countries found a correlation between TOP and DV, relating to physical, emotional and sexual violence. Reported prevalence of DV varied from 7-29\% (recent) and $21-39.5 \%$ (lifetime). Rates were higher amongst women who did not tell their partners about the TOP. There was a consistent correlation with DV and multiple terminations. The reasons that women in violent relationships chose to terminate was examined: two studies found women who were pressurised or forced by their partner into TOP. Descriptions of contraceptive sabotage and raperelated pregnancy were also identified. Five studies using male subjects found similar results.

Conclusion DV is common among women seeking TOP. Their healthcare and safety needs differ from the general population and should be considered by care providers.

\section{PP.54 WITHDRAWN BY AUTHOR}

\section{PP.55 SCREENING WOMEN FOR ASYMPTOMATIC BACTERIURIA IN EARLY PREGNANCY MAY REDUCE THE RISK OF LOW BIRTH WEIGHT}

doi:10.1136/archdischild-2013-303966.332

${ }^{1} \mathrm{HE}$ Browne, 'EA Bonney, ${ }^{2} \mathrm{LA}$ McDowell, ${ }^{2} \mathrm{~J}$ Young, ${ }^{1} \mathrm{NAB}$ Simpson. 'Division of Clinical Sciences, Leeds Institute of Molecular Medicine, University of Leeds, Leeds, UK; ${ }^{2}$ School of Medicine, University of Leeds, Leeds, UK

Introduction Anatomical changes in pregnancy predispose women to asymptomatic bacteriuria (ASB). Current NICE guidance in the UK advises that a mid-stream urine (MSU) sample should be offered in early pregnancy to screen for and treat ASB.

Aims To investigate how many women currently have an MSU in early pregnancy ( $<18$ weeks gestation) in an inner-city maternity unit; to evaluate whether current screening practise affects pregnancy outcome.

Methods This was a retrospective cohort study which examined notes of all delivered women who gave birth within a three-week period. Appropriate parametric and non-parametric tests were used to determine intergroup differences.

Results 243 women delivered during this period. Although all had been booked before 18 weeks gestation, only $9 \%(n=22)$ had been screened for ASB. Of the unscreened population, 18\% $(n=40)$ subsequently developed a UTI in pregnancy, and these were more likely to deliver a LBW baby $\left(18 \%<2500 \mathrm{~g}\right.$; OR $2.9,95^{\text {th }}$ CI $\left.1.1-7.9\right)$. The screened population had rates of LBW (4\%) which were the same as the unaffected cohort within the unscreened population $(6 \%)$.

Conclusion These data suggest that a policy of not screening for ASB may increase the chance of LBW babies being born. Although other confounding variables were not controlled for in this study, the evidence would suggest a policy of screening for ASB remains a reasonable option in pregnancy. (supported by Cerebra)

Keywords Asymptomatic bacteriuria/pyelonephritis/preterm labour/ low birth-weight.

\section{PP.56 ABNORMAL PLACENTAL PATHOLOGY AND SHORT-TERM NEONATAL OUTCOMES IN THE PRETERM INFANT}

doi:10.1136/archdischild-2013-303966.333

'EA Bonney, ${ }^{1} \mathrm{HE}$ Browne, ${ }^{2} \mathrm{~L}$ Barrett, ${ }^{2} \mathrm{C}$ Coulter, ${ }^{2} \mathrm{~J}$ Morris, ${ }^{4} \mathrm{DC}$ Greenwood, ${ }^{3} \mathrm{~J}$ Stahlschmidt, ${ }^{1} \mathrm{JJ}$ Walker, ${ }^{1} \mathrm{NAB}$ Simpson. 'Division of Clinical Sciences, Leeds Institute of Molecular Medicine, University of Leeds, Leeds, UK; ${ }^{2}$ School of Medicine, University of Leeds, Leeds, UK; ${ }^{3}$ Department of Histopathology, Leeds Teaching Hospitals NHS Trust, Leeds, UK: ${ }^{4}$ Centre for Epidemiology and Biostatistics, University of Leeds, Leeds, UK

Introduction Placental pathology provides a unique insight into the intrauterine environment prior to preterm birth

Objectives To investigate correlations between maternal variables and abnormal placental pathology in a cohort of women delivering extremely preterm; to determine associations between these and the incidence of adverse short-term neonatal outcome. 
Method Placental histopathology reports from women who gave birth in Leeds between 22-32 weeks gestation from 2009-2011 were obtained. Relevant maternal and neonatal data were accessed. Intergroup differences and trends were sought using Mann-Whitney $U$ and logistic/linear regression analyses where appropriate.

Results 269 women were included in the study. $89 \%$ of the placentas demonstrated abnormal pathology (infection/inflammation and/or ischaemia/infarction). Smoking and socioeconomic deprivation were associated with an increased incidence of placental infection $(p=0.02)$ and ischaemia $(p=0.0001)$. Babies were born significantly earlier if their placenta demonstrated infection than those whose showed ischaemia $(p=0.0001)$. Neonates born with infected placentas tended to be more likely to have intraventricular haemorrhage than those with ischaemic placentas (adjusted OR 1.9; 95 ${ }^{\text {th }}$ CI $0.5-6.5, \mathrm{p}=0.3$ ).

Conclusion Intrauterine infection/inflammation is the predominant antecedent of extreme prematurity, and is associated with maternal smoking and socioeconomic deprivation. Further studies need to consider the mechanisms which link these features. (supported by Cerebra)

\section{PP.57 FETAL MICROCHIMERISM DOES NOT APPEAR TO BE IMPLICATED IN EPITHELIAL OVARIAN CANCER}

doi:10.1136/archdischild-2013-303966.334

KL Liang, U Mahmood, K O'Donoghue. Department of Obstetrics and Gynaecology, Cork University Maternity Hospital, University College Cork, Cork, Ireland

Objective Ovarian cancer is the second most common gynaecologic cancer, and epithelial ovarian tumours are the most common malignant ovarian tumours. Pregnancy is an important factor in risk reduction and improved survival in epithelial ovarian cancers. Persistence of fetal cells after pregnancy, or fetal microchimerism, has been implicated in some female malignancies such as cervical and breast with different hypothesised roles. In this retrospective study, we aimed to determine if fetal microchimeric cells were involved in the progression of ovarian cancer.

Study design We investigated a well-characterised archive of epithelial ovarian tumour paraffin-embedded tissue sections from nulliparous and parous women. Fluorescence in situ hybridisation (FISH) was performed to detect male presumed-fetal cells. The outcome of blinded FISH analysis was correlated with reproductive history and clinic-pathological features of the cohort.

Results Fetal microchimeric cells were not detected in archived ovarian tumour formalin-fixed and paraffin-embedded tissue sections from parous women. Male-presumed fetal cells were instead found in sections from two nulliparous women, one of who had a previous miscarriage. Tumour cells were found to have multiple copies of X chromosomes by FISH analysis, and some tumour cells had loss of X chromosome relative to the ploidy level.

Conclusion These findings imply that fetal microchimerism does not have a significant role in the progression of ovarian cancer. Instead, fetal microchimerism could have a role in preventing the development of ovarian cancer by sensitising the maternal immune system to develop adaptive immunity against tumour cells that express onco-fetal antigens.

\section{PP.58 OSTEOPATHIC MANIPULATIVE TREATMENT DURING LABOUR: AN EXPLORATORY STUDY}

doi:10.1136/archdischild-2013-303966.335

1.2P Turi, ${ }^{2,3} \mathrm{G}$ Pizzolorusso, ${ }^{1} \mathrm{~A}$ Di Matteo. ${ }^{1}$ University G. D'Annunzio, Chieti, Italy; ${ }^{2} E B O M$, European Institute for Evidence Based Osteopathic Medicine, Chieti, Italy; ${ }^{3} \mathrm{AlOT}$, Accademia Italiana Osteopatia Tradizionale, Pescara, Italy
Objective Osteopathic manipulative treatment (OMT) is an alternative medical practise that focuses on body health by treating the musculoskeletal framework. Its aim is to affect positively the body's nervous, circulatory and lymphatic systems [1].

To date, the use of OMT during labour has been limited [2,3], although its effects are known during pregnancy $[4,5]$.

Aim of this exploratory study is to investigate the effect of OMT in a group of women during labour.

Methods Of $\mathrm{N}=119$ women enrolled and in addition to ordinary care, $\mathrm{N}=57$ received OMT. Endpoints were differences in vaginal tears, Apgar scores and umbilical artery $\mathrm{pH}$ levels.

Statistical analysis was based on multivariate regressions, focusing on the independent effect of OMT on primary outcomes.

Results Baseline characteristics were balanced in both groups. OMT ( $\beta-0.589 ; 95 \%$ CI -0.841 to $-0.337 ; p<0.0001)$ and episiotomy $(\beta-1.053 ; 95 \%$ CI -1.334 to -0.773 ; $p<0.0001)$ were significantly associated with a reduction in vaginal tears.

Only OMT was significantly associated to higher Apgar $1 \mathrm{~min}$ $(\beta 0.572 ; 95 \%$ CI 0.240 to $0.903 ; p<0.001)$ and 5 min scores $(\beta 0.465$; $95 \%$ CI 0.240 to $0.689 ; \mathrm{p}<0.0001$ ).

Risks factors for umbilical pH levels $<7.35$ were OMT (OR 0.363; $95 \%$ CI 0.149 to $0.848 ; p=0.02)$ and women at second delivery (OR 0.346; 95\% CI 0.129 to 0.918 ; $p=0.03$ ).

Conclusions OMT during delivery was found to affect positively vaginal tears, Apgar scores and umbilical $\mathrm{pH}$ levels.

\section{REFERENCES}

1. Van Buskirk RL. Nociceptive reflexes and the somatic dysfunction: a model. J Am Osteopath Assoc 1990;792-4, 797-809.

2. Whiting LM. Can the length of labour be shortened by osteopathic treatment? J Am Osteopath Assoc 1911;11:917-921.

3. Hart LM. Obstetrical practise. J Am Osteopath Assoc 1918:609-614.

4. Licciardone JC, Buchanan S, Hensel KL, King HH, Fuld KG, Stoll ST. Osteopathic manipulative treatment of back pain and related symptoms during pregnancy: a randomised controlled trial. Am J Obstet Gynecol 2010;202:43

5. King HH, Tettambel MA, Lockwood MD, Johnson KH, Arsenault DA, Quist R. Osteopathic manipulative treatment in prenatal care: a retrospective case control design study. J Am Osteopath Assoc 2003;103:577-582.

\section{PP.59 DEVELOPMENT AND PILOT OF HAND HELD PRE-PREGNANCY DIABETES NOTES IN ENGLAND}

doi:10.1136/archdischild-2013-303966.336

${ }^{1 P}$ Brydon, ${ }^{1 S}$ Clifford, ${ }^{2} \mathrm{~T}$ Smyth, ${ }^{3} \mathrm{~J}$ Webb, ${ }^{4} \mathrm{~J}$ Dhallu, ${ }^{1} \mathrm{~S}$ Giddings, ${ }^{1} \mathrm{~K}$ Morse, ${ }^{5} \mathrm{~N}$ Shah,

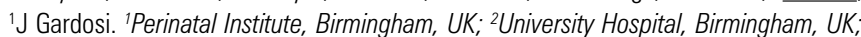
${ }^{3}$ Heart of UK NHS Foundation Trust, Birmingham, UK; ${ }^{4}$ Sandwell and West Birmingham NHS Trust, Birmingham, UK; ${ }^{5}$ Maternal - Fetal Medicine, City Hospital, Birmingham, UK

Background The majority of expectant mothers with diabetes fail to achieve the recommended glycaemic control prior to pregnancy. ${ }^{1}$ Suboptimal preparation for pregnancy is known to be associated with adverse outcomes. ${ }^{2}$ Our aim was to develop handheld notes to standardise and enhance pre-pregnancy care for women with preexisting diabetes.

Methods A multi-professional group designed the record along national recommendations ${ }^{3}$ to encourage early engagement and enhance communication between primary and secondary care. The 6 month pilot of 160 notes was conducted in 13 hospitals and 5 primary care centres.

Results The notes are an 8 page document ${ }^{4}$ and include a traffic light system to alert women of the preparations needed before starting a pregnancy. They contain prompts and checklists for clinicians to cover the essential information and management plans. Participants included women with type 1 and type 2 diabetes. The majority of respondents were new to preconception services. All participants thought that the notes were very useful in highlighting preparation and control prior to pregnancy. Two thirds felt the 